\title{
ANÁLISE DE ATITUDES DE ALUNOS UNIVERSITÁRIOS EM RELAÇÃO À ESTATIÍSTICA POR MEIO DA TEORIA DE RESPOSTA AO ITEM
}

\author{
Analysis of attitudes of university students in relation \\ to statistics through the Theory of Response to item
}

\author{
Claudia Turik ${ }^{1}$. Lori Viali² • João Feliz Duarte de Moraes $^{3}$
}

\begin{abstract}
Resumo: O presente estudo faz uma análise das respostas fornecidas por uma amostra de 360 graduandos à Escala de Atitudes em relação à Estatística (EAE). Para tanto, o ajuste do Modelo de Resposta Gradual da Teoria de Resposta ao Item foi efetuado no software PARSCALE. A escala apresentou parâmetros de discriminação dos itens $\left(a_{i}\right)$ indo de moderado a alto. Os parâmetros de dificuldade dos itens $\left(\mathrm{b}_{\mathrm{i}}\right)$ mostraram que a proposição de mais fácil concordância por parte dos alunos foi referente à tensão sentida, pelos mesmos, durante as aulas de estatística. As variáveis que mostraram influenciar no nível de atitudes foram: área de estudo, autopercepção de desempenho na disciplina e reconhecimento da aplicabilidade da estatística. Esses resultados sugerem que sejam adotadas estratégias para desenvolvimento de um ambiente de ensino-aprendizado estimulante, agradável, e que reforce as aplicações da estatística no contexto do curso de cada estudante.
\end{abstract}

Palavras-chave: Educação estatística. Teoria de Resposta ao Item.

\begin{abstract}
In this study we analyze the responses provided by a sample of 360 students at a private university in a Scale of Attitudes towards Statistics (EAE) test. The fit of the Graded Response Model of the Item Response Theory was made using the software PARSCALE. The scale showed the discrimination of items ( $\left.a_{i}\right)$ going from moderate to high level. The parameters of difficulty of the items $\left(b_{\text {. }}\right.$ ) showed that the proposition of easy agreement on the part of students was effected by the tension felt doing statistics classes. The variables that showed significance in the level of influence attitudes were: area of study, self-perception of performance in the discipline and the applicability of statistics. These results suggest that strategies have to be adopted to develop an environment for teaching and learning that is exciting and enjoyable, and to enhance the applications of statistics in the context of the subject for each student.
\end{abstract}

Keywords: Statistics Education. Item Response Theory.

\footnotetext{
${ }^{1}$ Instituto Federal de Educação, Ciência e Tecnologia do Rio Grande do Sul (IFRS). Rua Eng. Alfredo, 475, Centro. Rio Grande, RS, Brasil. 96.201-460. claudia.turik@riogrande.ifrs.edu.br

${ }^{2}$ Programa de Pós-Graduação em Educação em Ciências e Matemática, Pontifícia Universidade Católica do Rio Grande do Sul. Porto Alegre, RS, Brasil.

${ }^{3}$ Departamento de Estatística, Universidade Federal do Rio Grande do Sul. Porto Alegre, RS, Brasil.
} 


\section{Introdução}

Em muitos estudos da área educacional o interesse recai sobre variáveis que não podem ser observadas diretamente por se tratarem de características implícitas pessoais. Variáveis dessa natureza são chamadas de traços latentes. Não sendo observável, o traço latente necessita ser inferido com base em variáveis secundárias, observáveis, e que estejam relacionadas a ele. Para isso, frequentemente é utilizado um conjunto de itens que compõem um instrumento de medida.

No contexto da educação estatística, o conjunto de atitudes dos alunos com relação a esta disciplina tem se mostrado uma variável significativa para a compreensão dos fatores que influenciam o processo de ensino-aprendizagem. Um estudante com atitudes positivas em relação à disciplina de estatística tem maior probabilidade de sucesso na sua aprendizagem. Se apresentarmos atitudes favoráveis ao objeto de estudo, provavelmente, estaremos mais motivados para a aprendizagem (BRAGHIROLLI; PEREIRA; RIZZON, 1999).

As atitudes representam um esquema mental, não observável, que atua como mediador entre o pensamento e o comportamento. Trata-se, portanto, de um traço latente, o qual pode ser estimado a partir de instrumentos de medida, como a Escala de Atitudes em Relação à Estatística (EAE) (CARZOLA et al., 1999).

Pasquali (2003) coloca que a modelagem estatística utilizada na estimação de traços latentes, chamada de Teoria Clássica dos Testes (TCT), apresenta algumas restrições quanto a sua aplicação para medidas de outros traços que não aptidões, o caso da medida de atitudes, onde não existem respostas certas ou erradas. A TCT é centrada na explicação do resultado final de um teste dado pela soma dos escores brutos obtidos em seus itens. Esta teoria apresenta limitações que podem ser solucionadas pela Teoria de Resposta ao Item (TRI), também chamada de teoria psicométrica moderna.

A Teoria de Resposta ao Item foi construída a partir da ideia de traços latentes. Ela é composta de uma família de modelos matemáticos que fornecem a probabilidade de determinada resposta a um item ser escolhida. A resposta depende dos parâmetros que caracterizam o item e do nível do respondente quanto ao traço latente que está sendo medido. No caso de uma escala de atitudes, a TRI procura formas de representar a relação entre a probabilidade de um indivíduo concordar com um item, as características do item e a atitude enquanto traço latente, variando de mais favorável para mais desfavorável. O processo psíquico das atitudes do sujeito é a causa da expressão comportamental indicada por este na escolha da resposta ao item.

O impacto causado pela TRI se deve ao fato de ela superar várias limitações da Teoria Clássica dos Testes, de modo que:

1 O cálculo dos parâmetros de dificuldade e discriminação de um item passa a ser independente da amostra que foi utilizada;

2 A avaliação do traço latente dos sujeitos passa a ser independente do instrumento utilizado para medi-la;

3 Cada item é analisado em particular, independentemente do restante dos itens que compõem o instrumento de medida;

$4 \mathrm{O}$ traço latente passa a ser mensurado com maior precisão, pois são atribuídos pesos diferentes para itens com características distintas.

5 A TRI oferece uma medida de precisão para cada nível de traço latente (informação do teste). 
Análise de atitudes de alunos universitários ...

Este estudo tem por objetivo analisar as atitudes dos estudantes do terceiro grau em relação à estatística por meio da TRI, explorando os diferentes aspectos possibilitados pelo uso desta teoria.

Escala de Atitudes em Relação à Estatística (EAE): A escala é do tipo Likert e sua composição compreende vinte itens, dez relacionados a atitudes positivas e dez relacionados a atitudes negativas em relação à estatística. Cada item conta com quatro alternativas: discordo totalmente (um), discordo (dois), concordo (três) e concordo totalmente (quatro).

Modelo de Resposta Gradual: O modelo da TRI utilizado neste estudo para a análise dos dados foi o Modelo de Resposta Gradual (MRG), proposto por Samejima (1969). Ele é adequado para analisar os dados da Escala de Atitudes em relação à Estatística, pois a mesma é composta de itens com respostas politômicas e ordenadas.

Supondo que os escores das categorias de resposta de um item $i$ estejam dispostos em ordem crescente, e denotando por $k=0,1,2, \ldots, m_{i}$ onde $\left(m_{i}+1\right)$ é o número de categorias do i-ésimo item, a probabilidade de um indivíduo $j$ escolher uma determinada categoria $k$ no item $i$ pode ser dada pela equação (1).

$$
\begin{aligned}
& \mathrm{P}_{\mathrm{i}, \mathrm{k}}\left(\theta_{\mathrm{j}}\right)=\frac{1}{1+\mathrm{e}^{-\mathrm{Da}_{\mathrm{j}}\left(\theta_{\mathrm{j}}-\mathrm{b}_{\mathrm{i}, \mathrm{k}}\right)}}-\frac{1}{1+\mathrm{e}^{-\mathrm{Da}_{\mathrm{j}}\left(\theta_{\mathrm{j}}-\mathrm{b}_{\mathrm{i}, \mathrm{k}+1}\right)}} \\
& \mathrm{Com}: i=1,2, \ldots, i \quad j=1,2, \ldots, n \quad k=0,1,2, \ldots, m_{i} \quad b_{i, 1}<b_{i, 2}<\ldots<b_{i, m}
\end{aligned}
$$

Onde:

$i$ : Número de itens do teste;

$j:$ Número de respondentes;

$\theta$ : Atitudes em relação à estatística do j-ésimo indivíduo (traço latente);

$a_{i}$ : Parâmetro discriminação do item $i$. Representa a capacidade do item de diferenciar indivíduos com diferentes níveis do traço latente.

$b_{i, k}:$ Parâmetro de dificuldade da k-ésima categoria do item $i$. Pode ser particionado em dois termos, sendo $b_{i}$ o parâmetro de dificuldade geral do item. Um item é considerado tanto mais difícil quanto maior for o nível de traço latente que o sujeito necessita apresentar para aceitá-lo;

$D$ : Constante equivalente a 1 para a curva logística ou a 1,7 para obter a aproximação para a distribuição normal.

\section{Método}

A amostra foi constituída de trezentos e sessenta alunos, regularmente matriculados em uma disciplina de estatística de uma universidade particular gaúcha no segundo semestre de 2006. Os participantes responderam à Escala de Atitudes em relação à Estatística (EAE) de Carzola et al. (1999) e a um questionário composto de oito questões que visavam obter informações sobre os mesmos. A participação foi opcional e precedida de uma explicação a respeito do caráter não avaliativo da referida pesquisa.

Para proceder à análise via TRI, foi utilizado o software PARSCALE (2003). 


\section{Resultados e discussão}

A idade dos alunos da amostra variou entre 17 e 56 anos, com média de 23,59 e desvio-padrão de 6,89 anos. Com relação ao gênero, 42,58\% dos entrevistados foram do gênero masculino e $57,42 \%$ do gênero feminino. Quanto à ocupação, 37,99\% dos avaliados estavam apenas estudando, 28,21\% eram estagiários e 33,80\% exerciam algum trabalho remunerado. A maior parte dos alunos estava cursando a disciplina pela primeira vez, porém 18,44\% já haviam sido reprovados na disciplina, cancelado ou desistido de cursá-la antes do seu término em semestres anteriores.

Os entrevistados foram agrupados de acordo com a respectiva área de conhecimento do seu curso. As frequências são apresentadas na Tabela 1.

Tabela 1. Frequências por área de conhecimento

\begin{tabular}{llrc}
\hline \multicolumn{1}{c}{ Área do conhecimento } & \multicolumn{1}{c}{ Cursos selecionados } & Alunos & Percentual (\%) \\
\hline Ciências Sociais Aplicadas & Administração de empresas, relações públicas. & 160 & 44,4 \\
Ciências Biológicas & Biologia & 63 & 17,5 \\
Ciências Humanas & Educação, psicologia, geografia. & 106 & 29,4 \\
Ciências Exatas & Engenharia & 31 & 8,6 \\
Total & & 360 & 100,0 \\
\hline
\end{tabular}

Fonte: Dados de pesquisa.

Uma minoria dos alunos acredita não haver nenhuma aplicação da estatística no cotidiano $(8,06 \%)$, porém, quando estes são tomados em conjunto com os alunos que consideram que exista pouca aplicação da estatística em problemas reais, este percentual chega a 52,22\% de alunos que não percebem uma grande contribuição prática do aprendizado de estatística. Em relação ao desempenho na disciplina de estatística, $24,44 \%$ dos alunos o consideraram fraco, $37,78 \%$ regular e $37,78 \%$ bom.

A pontuação média, em termos de escore bruto (TCT), alcançada na Escala de Atitudes em Relação à Estatística foi de 47,91 pontos (desvio-padrão $=11,20$ ), variando de vinte a oitenta pontos. Tomando o ponto médio da escala (cinquenta pontos) como ponto de corte, tem-se que 56,39\% dos alunos apresentaram atitudes desfavoráveis, enquanto 43,61\% apresentaram atitudes favoráveis em relação à estatística.

Para a aplicação do Modelo de Resposta Gradual de Samejima (1969) da TRI, foram verificados seus pressupostos teóricos do MRG, a saber: unidimensionalidade e independência local.

A dimensionalidade da EAE foi verificada com uma análise fatorial exploratória da escala (método dos fatores principais), rotação ortogonal varimax, adotando-se, para a extração dos fatores, o critério de autovalores maiores que um. O modelo produziu dois fatores relacionados aos vinte itens da escala utilizada. O modelo obtido explicou $64,05 \%$ da variância total, com o primeiro fator responsável por $53,02 \%$ da explicação da variância total, e o segundo, por $11,03 \%$. A estatística KMO (Kaiser-Meyer-Olkin), utilizada como medida de ajuste da amostra foi de 0,96 e o Teste de Esfericidade de Bartlett forneceu uma estatística de 
teste, $\chi^{2}$, significativa ao nível de 0,01 , indicando que a técnica de análise fatorial estava adequada para analisar a escala.

A Tabela 2 mostra que foram encontrados dois fatores relacionados às atitudes: o primeiro com itens relacionados a atitudes favoráveis e o segundo relacionado a atitudes desfavoráveis.

Tabela 2. Cargas fatoriais dos itens

\begin{tabular}{|c|c|c|}
\hline \multirow[b]{2}{*}{ Itens } & \multicolumn{2}{|c|}{ Cargas fatoriais } \\
\hline & Fator 1 & Fator 2 \\
\hline Eu gosto realmente de estatística. & 0,81 & - \\
\hline A estatística me faz sentir seguro (a) e é, ao mesmo tempo, estimulante. & 0,81 & - \\
\hline A estatística é fascinante e divertida. & 0,81 & - \\
\hline A estatística é uma das matérias que eu realmente gosto de estudar na faculdade. & 0,79 & - \\
\hline $\begin{array}{l}\text { Eu tenho uma reação definitivamente positiva com relação à estatística: Eu gosto e } \\
\text { aprecio essa matéria. }\end{array}$ & 0,78 & - \\
\hline A estatística é algo que eu aprecio grandemente. & 0,75 & - \\
\hline Eu me sinto tranquilo (a) em estatística e gosto muito dessa matéria. & 0,72 & - \\
\hline Eu fico mais feliz na aula de estatística do que na aula de qualquer outra matéria. & 0,72 & - \\
\hline Eu acho a estatística muito interessante e gosto das aulas de estatística. & 0,72 & - \\
\hline O sentimento que tenho com relação à estatística é bom. & 0,55 & - \\
\hline $\begin{array}{l}\text { "Dá um branco" na minha cabeça e não consigo pensar claramente quando estudo } \\
\text { estatística. }\end{array}$ & - & 0,80 \\
\hline Eu tenho sensação de insegurança quando me esforço em estatística. & - & 0,79 \\
\hline $\begin{array}{l}\text { A estatística me faz sentir como se estivesse perdido (a) em uma selva de números } \\
\text { e sem encontrar a saída. }\end{array}$ & - & 0,78 \\
\hline Pensar sobre a obrigação de resolver um problema estatístico me deixa nervoso (a). & - & 0,75 \\
\hline $\begin{array}{l}\text { Eu encaro a estatística com um sentimento de indecisão, que é resultado do medo } \\
\text { de não ser capaz em estatística. }\end{array}$ & - & 0,73 \\
\hline A estatística me deixa inquieto (a), descontente, irritado (a) e impaciente. & - & 0,73 \\
\hline Eu nunca gostei de estatística e é a matéria que me dá mais medo. & - & 0,69 \\
\hline Eu fico sempre sob uma terrível tensão na aula de estatística. & - & 0,68 \\
\hline Eu não gosto de estatística e me assusta ter que fazer essa matéria. & - & 0,65 \\
\hline Quando eu ouço a palavra estatística, eu tenho um sentimento de aversão. & - & 0,62 \\
\hline
\end{tabular}

Fonte: Dados de pesquisa.

O diagrama de declividade (scree plot) possibilita a visualização gráfica dos dois fatores com autovalores acima de um (Figura 1).

Para satisfazer o postulado da unidimensionalidade, é suficiente admitir que haja uma dimensão dominante no conjunto de itens (PASQUALI, 2003), o caso do modelo obtido, que forneceu um fator principal responsável por $53,02 \%$ da explicação da variância total. Visto que o teste pode ser considerado unidimensional, assume-se que somente as atitudes em relação à estatística constituem um fator de influência nas respostas dos itens, determinando a independência local.

O parâmetro $\theta$, que representa o traço latente, pode assumir, teoricamente, qualquer valor entre - $\infty$ e $+\infty$. É preciso, portanto, estabelecer uma origem e uma unidade de medida para definição da escala de atitudes. Estes valores são determinados de forma a representar, respectivamente, a média e o desvio-padrão das atitudes dos alunos em relação à estatística. No presente estudo usou-se uma escala com média igual à zero $(\mu=0)$ e desvio-padrão igual 
Turik, C.; Viali, L.; Moraes, J. F. D.

a um $(\theta=1)$. Vale ressaltar que os valores referentes ao nível de atitudes em relação à estatística não possuem um significado prático, o importante são as relações existentes entre seus pontos. As estimativas dos parâmetros do traço latente variaram de -3,00 a 3,34, distribuídos de acordo com a Figura 2.

Figura 1. Diagrama de declividade.

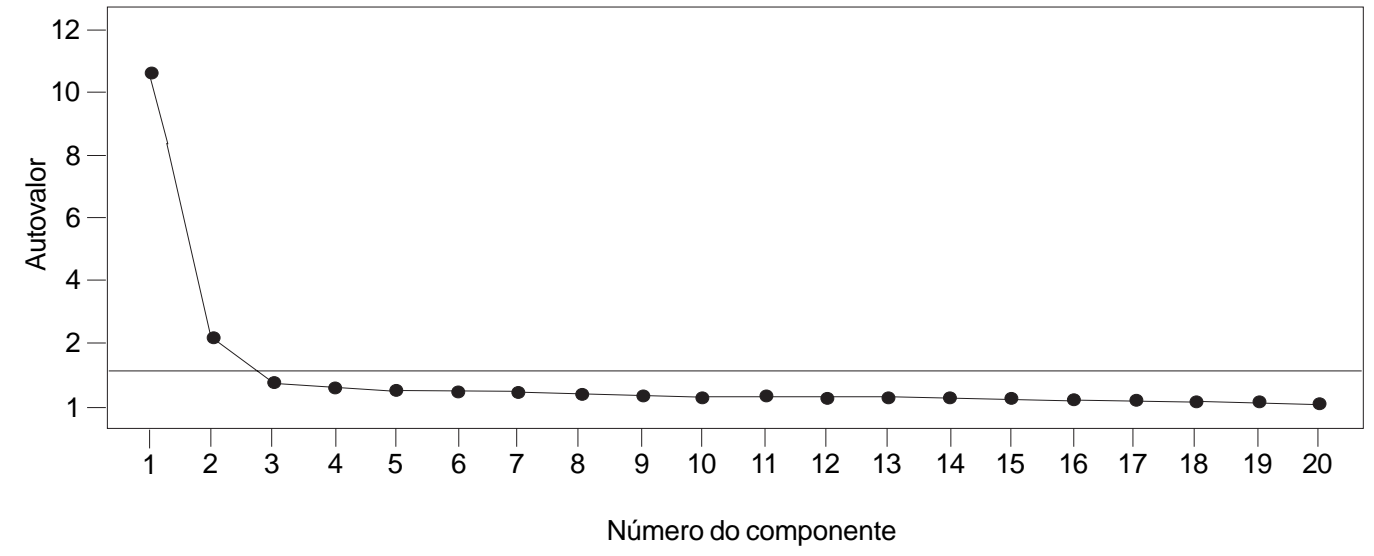

Fonte: Dados de pesquisa.

Figura 2. Histograma dos escores latentes.

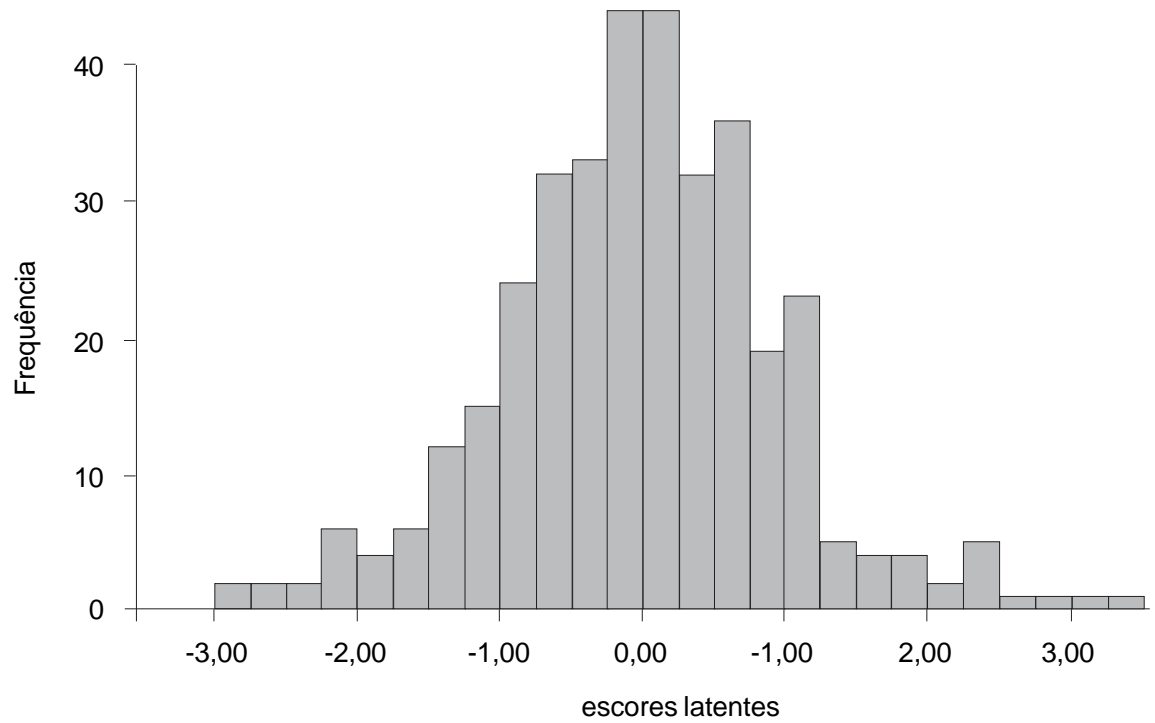

Fonte: Dados de pesquisa. 
Análise de atitudes de alunos universitários ...

Uma das características que refletem a qualidade de um item é o seu poder de discriminar sujeitos com magnitudes distintas no traço latente avaliado. A investigação de cada um dos itens da EAE mostrou os parâmetros de discriminação (a-) variando entre 0,81 (item 1) e 2,24 (item 20). De acordo com as categorias sugeridas por Baker (2001 apud ALBUQUERQUE; TRÓCCOLI, 2004), o nível de discriminação dos itens variou de moderado a muito alto (Tabela 3).

Tabela 3. Classificação dos itens da EAE de acordo com seus índices de discriminação

\begin{tabular}{lllc}
\hline \multicolumn{1}{c}{ Discriminação } & Pontos de corte & \multicolumn{1}{c}{ Itens } & \% de itens \\
\hline Moderada & 0,65 a 1,34 & $1,2,6,7,8,10,13,16,17$ & 45 \\
Alta & 1,35 a 1,69 & $3,4,5,9,11,12,15,18$ & 40 \\
Muito alta & 1,70 ou mais & $14,19,20$ & 15 \\
\hline
\end{tabular}

Fonte: Dados de pesquisa.

Os itens 14 (Eu gosto realmente de estatística), 19 (Eu me sinto tranquilo (a) em estatística e gosto muito dessa matéria) e 20 (Eu tenho uma reação definitivamente positiva com relação à estatística: Eu gosto e aprecio essa matéria) são aqueles que melhor discriminam a população quanto ao nível de atitudes em relação à estatística.

Os itens 1 (Eu fico sempre sob uma terrivel tensão na aula de estatística), 7 (Eu tenho sensação de insegurança quando me esforço em estatística) e 10 (A estatística me faz sentir como se estivesse perdido(a) em uma selva de números e sem encontrar a saída) são os que menos discriminam a população quanto ao traço latente.

As estimativas do parâmetro de dificuldade do item $\left(\mathrm{b}_{\mathrm{i}}\right)$ estão na mesma escala que as estimativas do traço latente $(\theta)$. Os valores obtidos apontam que o item 18 (Eu fico mais feliz na aula de estatística do que na aula de qualquer outra matéria) é o que requer atitudes mais intensamente favoráveis para que o aluno esteja de acordo com ele $\left(b_{i}=2,24\right)$. A Figura 3 mostra quatro curvas, representando cada uma das quatro categorias de resposta possíveis para o item 18. Tais curvas são chamadas de Curvas Características do Item e mostram a relação, dada pela equação (1), entre a probabilidade de escolha em cada categoria de resposta e o nível de atitudes em relação à estatística. Percebe-se que, mesmo indivíduos com um nível razoável de atitudes positivas frente à estatística, têm maior probabilidade de discordar do que de concordar com sua proposição: A probabilidade de concordância com o item 18 (curva 3) torna-se superior à probabilidade de discordância (curva 2) somente para indivíduos com traço latente $(\theta)$ superior a 1,2. Ainda, a probabilidade de um sujeito concordar plenamente com o item 18 é superior às probabilidades das demais categorias somente para indivíduos com traço latente extremamente alto $(\theta>3)$.

O motivo da dificuldade de concordância com esse item, por parte dos alunos, provavelmente se deve ao fato de os participantes cursarem outros cursos que não o de graduação em estatística, tendo outros interesses principais. Como forma de aproveitar a motivação dos alunos por outras disciplinas, é conveniente que os professores de estatística busquem integrar o conhecimento construído ao longo do semestre com as demais disciplinas que os alunos cursam, adaptando e contextualizando os conteúdos. 
Em situação oposta, o primeiro item (Eu fico sempre sob uma terrivel tensão na aula de estatística) foi com o que os alunos mais facilmente concordaram $\left(b_{i}=-0,56\right)$. Para reverter este quadro, os docentes devem procurar estabelecer um ambiente aprazível em sala de aula. Silva et al. (2002) colocam a importância da experiência agradável de aprendizagem no desenvolvimento das atitudes positivas em relação à estatística, e sugerem como meios de alcançá-la o uso de estratégias estimulantes e desafiadoras, com a utilização adequada de pacotes computacionais estatísticos.

Figura 3. Curva das categorias de resposta do item 18.

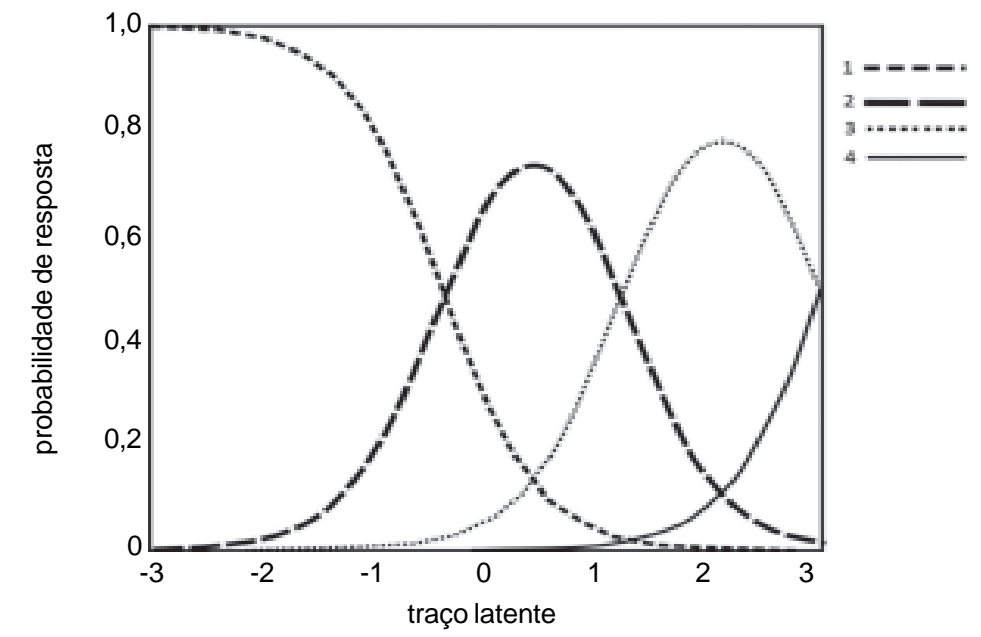

Fonte: Dados de pesquisa.

A Tabela 4 mostra os itens ordenados do mais fácil ao mais difícil. Percebe-se que os itens com que os alunos mais facilmente concordam remetem ao afeto negativo, enquanto os itens mais difíceis estão ligados ao afeto positivo.

Um teste é caracterizado pelos itens que o compõem. Os itens envolvidos devem ser suficientes para possibilitar que o instrumento seja informativo nos diferentes níveis da escala de escore latente. Considerando os vinte itens aos quais foi ajustado o Modelo de Resposta Gradual, analisou-se a Curva de Informação do Teste (Figura 4). A curva com linha contínua mostra que a EAE fornece maior quantidade de informações para alunos com intensidade de atitudes variando de $-2,00$ a 2,81. A curva com linha tracejada mostra que as atitudes são mais precisamente mensuradas, com menor erro, no mesmo intervalo, de $-2,00$ a 2,81. Dos sujeitos participantes deste trabalho, 3,33\% possuem traços latentes abaixo de - 2,00 e 0,83\% acima de 2,81 . 
Análise de atitudes de alunos universitários ...

Tabela 4. Índices de dificuldade dos itens ordenados

\begin{tabular}{lr}
\hline \multicolumn{1}{c}{ Itens } & b \\
\hline 01. Eu fico sempre sob uma terrível tensão na aula de estatística. & $-0,56$ \\
17. Eu nunca gostei de estatística e é a matéria que me dá mais medo. & $-0,49$ \\
02. Eu não gosto de estatística e me assusta ter que fazer essa matéria. & $-0,44$ \\
13. Eu encaro a estatística com um sentimento de indecisão, que é resultado do medo de não ser capaz & $-0,28$ \\
em estatística. & $-0,25$ \\
10. A estatística me faz sentir como se estivesse perdido(a) em uma selva de números e sem encontrar & $-0,24$ \\
a saída. & $-0,18$ \\
12. Quando eu ouço a palavra estatística, eu tenho um sentimento de aversão. & $-0,13$ \\
08. A estatística me deixa inquieto(a), descontente, irritado(a) e impaciente. & $-0,07$ \\
07. Eu tenho sensação de insegurança quando me esforço em estatística. & $-0,02$ \\
06. "Dá um branco" na minha cabeça e não consigo pensar claramente quanto estudo estatística. & 0,00 \\
09. O sentimento que tenho com relação à estatística é bom. & 0,09 \\
16. Pensar sobre a obrigação de resolver um problema estatístico me deixa nervoso(a). & 0,56 \\
03. Eu acho a estatística muito interessante e gosto das aulas de estatística. & 0,62 \\
14. Eu gosto realmente de estatística. & \\
20. Eu tenho uma reação definitivamente positiva com relação à estatística: Eu gosto e aprecio essa & 0,66 \\
matéria. & 0,67 \\
11. A estatística é algo que eu aprecio grandemente. & 0,75 \\
19. Eu me sinto tranquilo(a) em estatística e gosto muito dessa matéria. & 0,85 \\
05. A estatística me faz sentir seguro(a) e é, ao mesmo tempo, estimulante. & 0,86 \\
04. A estatística é fascinante e divertida. & 1,27 \\
15. A estatística é uma das matérias que eu realmente gosto de estudar na faculdade. & \\
18. Eu fico mais feliz na aula de estatística do que na aula de qualquer outra matéria. &
\end{tabular}

Fonte: Dados de pesquisa.

Figura 4. Curva de Informação do Teste.

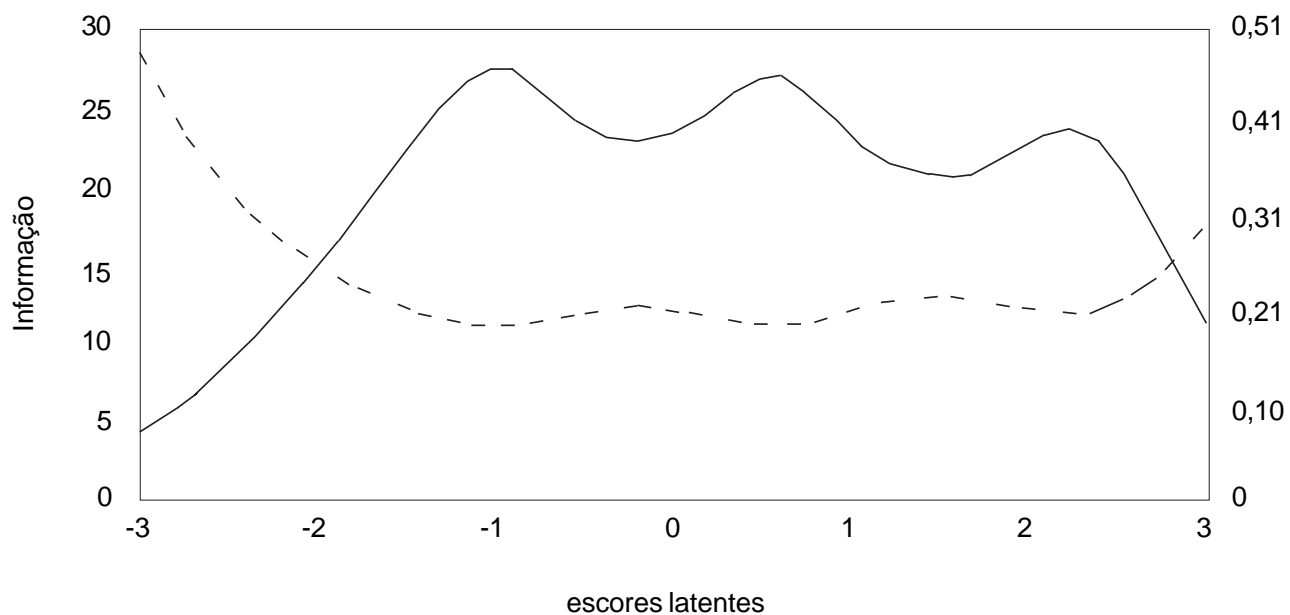

Fonte: Dados de pesquisa. 
Para verificar se a análise TRI fornece indícios de diferença no nível de atitudes em relação à estatística estimadas pelo escore latente entre grupos distintos de alunos, foram procedidas análises de variância. Nos casos em que foram detectadas diferenças, os dados foram submetidos ao teste post-hoc de Tukey. Os critérios para o agrupamento dos sujeitos (fatores) foram baseados nas respostas dadas ao Questionário do Aluno. Foram estes: diferentes gêneros, áreas de estudo, níveis de conhecimento da utilidade da estatística no cotidiano e níveis de desempenho autopercebido na disciplina de estatística. Os resultados estão dispostos na Tabela 5.

Tabela 5. ANOVA para as variáveis: número de créditos, aplicabilidade da estatística e percepção do desempenho (escores latentes)

\begin{tabular}{|c|c|c|c|c|c|}
\hline Variável & Respostas & Média & $\begin{array}{l}\text { Desvio- } \\
\text { padrão }\end{array}$ & $\mathbf{F}$ & $\mathbf{p}$ \\
\hline \multicolumn{6}{|l|}{ Gênero } \\
\hline & Feminino & $-0,02^{a}$ & 0,89 & 0,00 & 0,979 \\
\hline & Masculino & $-0,02^{a}$ & 1,06 & & \\
\hline \multicolumn{6}{|l|}{ Área de estudo } \\
\hline & Ciências sociais aplicadas & $-0,21^{a}$ & 0,97 & 6,34 & $<0,001$ \\
\hline & Ciências biológicas & $-0,12^{a b}$ & 0,80 & & \\
\hline & Ciências humanas & $0,22^{b}$ & 1,10 & & \\
\hline & Ciências exatas & $0,38^{\mathrm{b}}$ & 0,72 & & \\
\hline \multicolumn{6}{|c|}{ Aplicabilidade da estatística } \\
\hline & Não conheço nenhuma aplicação na realidade & $-0,75^{a}$ & 1,01 & 26,30 & $<0,001$ \\
\hline & Pouca aplicação em problemas reais & $-0,26^{b}$ & 0,89 & & \\
\hline & $\begin{array}{l}\text { Há muita aplicação no cotidiano } \\
\text { principalmente em pesquisas }\end{array}$ & $0,32^{\mathrm{c}}$ & 0,94 & & \\
\hline \multicolumn{6}{|c|}{ Percepção do desempenho } \\
\hline & Fraco & $-0,75^{a}$ & 0,90 & 60,70 & $<0,001$ \\
\hline & Regular & $-0,01^{b}$ & 0,78 & & \\
\hline & Bom & $0,53^{c}$ & 0,89 & & \\
\hline
\end{tabular}

Fonte: Dados da pesquisa.

Nota: Médias com letras diferentes diferem estatisticamente ao nível de $5 \%$ de significância - Teste de Tukey.

O agrupamento dos participantes revelou que não houve diferença significativa entre as atitudes dos estudantes do gênero masculino e feminino. Esses resultados são semelhantes aos das pesquisas de atitudes em relação à estatística, tais como as de Mendes (2003); Silva et al. (2002); Vendramini e Brito (2001); Quintino, Guedes e Martins (2001) e Silva (2000).

Quando as áreas de estudos são analisadas como fator de possível influência, percebe-se que os estudantes da área de ciências sociais aplicadas apresentaram atitudes mais desfavoráveis do que os alunos de ciências exatas e humanas $(F=6,74 ; p<0,001)$. Em média, as ciências exatas tiveram atitudes mais favoráveis em relação à estatística, seguidas das humanas, biológicas e ciências sociais aplicadas, porém as demais diferenças não foram estatisticamente significativas. As pesquisas anteriores não mostraram consenso em seus resultados, sendo que, por vezes, não foram verificadas diferenças significativas entre as áreas (VENDRAMINI; BRITO, 2001; QUINTINO; GUEDES; MARTINS, 2001), e, por vezes, a área de humanas 
Análise de atitudes de alunos universitários ...

foi apontada como tendo atitudes mais desfavoráveis (SILVA, 2000; SILVA et al., 2002). Um motivo a ser considerado para esta discrepância é o de que os critérios de escolha e agrupamento dos cursos de graduação em áreas de conhecimento são distintos conforme a pesquisa, fazendo com que uma mesma área de conhecimento seja representada por cursos diferentes ou com que um mesmo curso pertença a categorias diferentes de áreas do conhecimento.

O fato de a média das atitudes em relação à estatística dos alunos de exatas ser superior à dos demais pode ser consequência de um melhor relacionamento que estes alunos têm com a linguagem matemática. Existem livros com foco na parte conceitual da estatística, sem o uso de fórmulas matemáticas (DANCEY; REIDY, 2006; MAGNUSSON; MOURÃO, 2003), abordagem essa que pode ser adotada completa ou parcialmente por professores de disciplinas voltadas para os cursos de outras áreas científicas.

As atitudes dos alunos que reconhecem o uso da estatística no cotidiano tendem a ser mais positivas dos que as atitudes dos alunos que conhecem poucas aplicações, e estas mais positivas do que as atitudes dos alunos que desconhecem quaisquer aplicações ( $F=25,15 ; \mathrm{p}$ $<0,001)$. Esse resultado está de acordo com os estudos revisados que se propuseram a averiguar tal relação (SILVA, 2000; QUINTINO; GUEDES; MARTINS, 2001; VENDRAMINI; BRITO, 2001). Para reforçar a aplicabilidade da estatística, Vendramini e Brito (2001) sugerem que as aulas sejam iniciadas com a exposição de técnicas aplicadas e com a discussão de pesquisas já concluídas na área de interesse do estudante. A introdução dos conceitos teóricos da estatística ficaria para um segundo momento, visando o desenvolvimento de atitudes positivas dos alunos e consequente aumento da predisposição para o aprendizado.

A autopercepção dos alunos quanto a seu desempenho na disciplina é uma variável importante na análise das atitudes. O grupo de alunos que se percebe com bom desempenho também é aquele que possui atitudes mais positivas, da mesma forma que o grupo que se percebe com desempenho fraco tem atitudes significantemente menos positivas. ( $F=65,32 ; \mathrm{p}$ $<0,001)$. Este resultado é condizente com estudos anteriores (SILVA; CARZOLA; BRITO, 1999; QUINTINO; GUEDES; MARTINS, 2001; VENDRAMINI; BRITO, 2001; MENDES, 2003). As atitudes positivas dos estudantes podem ter influenciado no seu desempenho ao longo da disciplina, ao mesmo tempo em que um bom desempenho pode ter levado ao desenvolvimento de atitudes mais favoráveis.

\section{Considerações finais}

O ensino de estatística tem tido sua importância reconhecida, ao passo que sua prática se dá desde o Ensino Fundamental até grande parte dos cursos universitários. Em um âmbito geral, o aprendizado desta ciência é necessário para que a população tenha entendimento crítico das informações quantitativas que têm sido divulgadas por diversos canais de comunicação. De modo mais específico, os profissionais devem saber recorrer aos dados provenientes de pesquisas de suas áreas, para a busca de informação útil e confiável.

Instrumentos de avaliação como a EAE permitem um diagnóstico inicial das atitudes dos alunos de uma nova turma, possibilitando uma ação estratégica do professor no decorrer do curso. A melhoria das atitudes em relação à matéria influirá no ambiente da aula, no aprendizado dos alunos e na predisposição dos mesmos ao uso da estatística no futuro. 
Turik, C.; Viali, L.; Moraes, J. F. D.

O reconhecimento do estudante quanto à utilidade do conteúdo e a sua autopercepção de desempenho na disciplina são fatores que se mostram relacionados com as atitudes em relação à estatística. Como tal, estes devem ser explorados pelos docentes e instituições de ensino, favorecendo uma visão integrada e adaptável do ensino de estatística de acordo com o contexto de cada curso.

Um resultado relevante obtido com a análise psicométrica da EAE por meio do Modelo de Resposta Gradual foi a diversidade das características dos itens avaliados (parâmetros de discriminação e de dificuldade dos itens), indicando um ganho de precisão na aferição das atitudes por meio da TRI em relação à soma de escores brutos da TCT.

Entretanto, a Curva de Informação do Teste mostra que há necessidade de outros estudos testando a inclusão de novos itens visando obter um questionário que se configure como um instrumento com maior capacidade de discriminar estudantes com níveis de atitudes bastante baixos (atitudes desfavoráveis).

É importante a busca continuada por formas de desenvolver o ensino de estatística levando em conta os aspectos afetivos envolvidos neste processo. Vêm contribuir com tal objetivo, novos estudos que analisem possíveis fatores de influência nas atitudes em relação à estatística não explorada neste trabalho, tais como a opinião de amigos/parentes e características da instituição de ensino e do professor.

\section{Referências}

ALBUQUERQUE, A.; TROCCOLI, B. T. Desenvolvimento de uma escala de bem-estar subjetivo. Psicologia: teoria e pesquisa, Brasília, v. 20, n. 2, p. 153-164, ago. 2004.

BRAGHIROLLI, E.; PEREIRA, S.; RIZZON, L. A. Temas de psicologia social. 3. ed. Petrópolis: Vozes, 1999.

CAZORLA, I. M. et al. Adaptação e validação de uma escala de atitudes em relação à estatística. In: CONFERÊNCIA INTERNACIONAL EXPERIÊNCIAS E PERSPECTIVAS DO ENSINO DE ESTATÍSTICA, 1999, Florianópolis. Anais... Florianópolis: Universidade Federal de Santa Catarina, 1999. p. 45-57.

DANCEY, C. P.; REIDY, J. Estatística sem matemática para psicologia. 3. ed. Porto Alegre: ArtMed, 2006.

MAGNUSSON, W. E.; MOURÃO, G. Estatística sem matemática: a ligação entre as questões e a análise. Londrina: Ed. Planta, 2003.

MENDES, C. R. Atitude em relação a estatística e temas transversais: um estudo de caso. In: SEMINÁRIO IASI DE ESTATÍSTICA APLICADA, 9., 2003, Rio de Janeiro. Anais... Rio de Janeiro: Inter American Statistical Institute, 2003. v. 1. 1 CD-ROM.

PARSCALE for Windows. Version 4.1. Lincolnwood: Scientific Software International, 2003. [Computer program].

PASQUALI, L.; PRIMI, R. Fundamentos da teoria da resposta ao item. Avaliação

Psicológica, Porto Alegre, v. 2, n. 2, p. 99-110, dez. 2003. 
Análise de atitudes de alunos universitários ...

QUINTINO, C. A. A.; GUEDES, T. A.; MARTINS, A. B. T. Análise estatística das atitudes dos alunos de iniciação científica da Universidade Estadual de Maringá, em relação à disciplina Estatística - 2000. Acta Scientiarum, Maringá, v. 23, n. 6, p. 1523-1529, 2001.

SAMEJIMA, R. Estimation of latent ability using a response pattern of graded scores. Richmond: Psychometric Society, 1969. (Psychometric monograph, 17). Disponível em: < http://www.psychometrika.org/journal/online/MN17.pdf>. Acesso em: 10 jan. 2011.

SILVA, C. B. da. Atitudes em relação à estatística: um estudo com alunos de graduação. 2000. 157 f. Dissertação (Mestrado em Educação)-Faculdade de Educação, Universidade Estadual de Campinas, Campinas, 2000.

SILVA, C. B. da et al. Atitudes em relação à estatística e à matemática. PsicoUSF, Itatiba, v. 7, n. 2, p. 219-228, jul./dez. 2002.

SILVA, C. B. da.; CAZORLA, I. M; BRITO, M. R. F. de. Concepções e atitudes em relação à estatística. In: CONFERÊNCIA INTERNACIONAL EXPERIÊNCIAS E PERSPECTIVAS DO ENSINO DE ESTATÍSTICA, 1999, Florianópolis. Anais... Florianópolis: Universidade Federal de Santa Catarina, 1999. p. 18-29.

VENDRAMINI, C. M.; BRITO, M. R. F. de. Relações entre atitude, conceito e utilidade da Estatística. Psicologia Escolar e Educacional, João Pessoa, v. 5, n. 1, p. 59-63, jun. 2001. 